\title{
Changes in Heart Rate Variability in Patients With Atrial Fibrillation After Pulmonary Vein Isolation and Ganglionated Plexus Ablation
}

\author{
J. VESELA ${ }^{1}$, P. OSMANCIK ${ }^{1}$, D. HERMAN ${ }^{1}$, R. PROCHAZKOVA ${ }^{1}$ \\ ${ }^{1}$ Third Internal - Cardiology Clinic, University Hospital Kralovske Vinohrady, Charles University, \\ Prague, Czech Republic
}

Received July 10, 2017

Accepted July 10, 2018

Epub Ahead of Print October 23, 2018

\begin{abstract}
Summary
Pulmonary vein isolation (PVI) is the cornerstone in the treatment of patients with paroxysmal atrial fibrillation (PAF). Some research has suggested studies have shown that modification of ganglionated plexuses (GP), performed with PVI, could lead to even better outcomes. The aim of this study was to determine the effect of PVI on the autonomic system. Heart rate variability (HRV) was used as a marker of autonomic system activity. Twenty-six PAF patients underwent PVI (PVI group) and twenty patients underwent PVI plus a GP ablation (GP group). In each group, 5 min long ECG signals obtained before and after the electrophysiology EP study were analyzed. Time and frequency domain parameters were evaluated. Vagal responses during ablation were observed in 15 (58\%) patients in the PVI group and in $12(60 \%)$ patients in the GP group. The change in normalized power in the low frequency (LF) and in the LF/HF ratio, before and after ablation, was statistically significant in both groups (LF/HF 2.6 \pm 1.6 before vs. $1.4 \pm 1.7$ after ablation in PVI group and LF/HF 3.3 \pm 2.6 before vs. $1.8 \pm 1.9$ after ablation in the GP group). Relative to heart rate variability parameters, there were no differences between PVI and PVI + plus GP ablation.
\end{abstract}

\section{Key words}

Atrial fibrillation • Ablation • Heart rate variability • Ganglionated plexus

\section{Corresponding author}

P. Osmancik, Third Internal - Cardiology Clinic, Cardiocenter, University Hospital Kralovske Vinohrady, Srobarova 50, Prague, Czech Republic. Fax: 00420267162 817. E-mail: pavel.osmancik@gmail.com

\section{Introduction}

Atrial fibrillation (AF), the most common sustained arrhythmia, is associated with increased cardiovascular morbidity and mortality. Pulmonary vein isolation (PVI) is the cornerstone for treatment of patients with AF. In patients with paroxysmal AF (PAF), the success rate of PVI has been described as between $60-70 \%$ in the midterm (Wilber et al. 2010, Jaïs et al. 2008). However, despite the relatively good efficacy of PVI in PAF, the rate of arrhythmia reoccurrence remains high.

Autonomic tone seems to play an important role in the initiation of AF (Bettoni 2002). Recently, numerous studies have shown that radiofrequency energy applications at either proven or presumed autonomic ganglia (ganglionated plexuses, GP) locations, can significantly influence vagal reflexes and increase the efficacy of ablations (Pappone 2004). Therefore, modification of the left atrial GP has been proposed as a potential way to improve PAF treatment (Katritsis et al. 2011).

Heart rate variability (HRV) analysis is a very useful, non-invasive, investigative tool for evaluating changes in autonomic tone (Heart Rate Variability. Task Force of the European Society of Cardiology the North American Society of Pacing Electrophysiology 1996). HRV describes variations in both instantaneous heart rate and RR intervals. RR intervals are obtained from a surface ECG and subsequently analyzed using special software. Like targeted GP ablations, PVI can induce an immediate decrease in autonomic functions (Bauer et 
al. 2006). Calo et al. (2012) described that radiofrequency catheter ablation in the right atrium determined significant autonomic changes. Surprisingly, an assessment of HRV parameters following PVI performed with and without concomitant GP ablation has not been performed and therefore remains unknown. The aim of the study was to assess the effect of PVI and PVI plus GP ablation in the left atrium and on HR variability parameters (determined before and after catheter ablation). We hypothesized that PVI plus GP ablation would have more significant effect on HRV parameters compared to PVI alone.

\section{Methods}

\section{Patient population}

Patients with paroxysmal, or persistent AF were enrolled in the study. The definition of AF was based on current AHA/ACC/HRS guidelines (January et al. 2014). The study was performed as a prospective, observational study. Written informed consent was obtained from all participants, and the study was approved by the local Ethics Committee. All procedures performed in studies were in accordance with the ethical standards of the institutional research committee and with the 1964 Helsinki declaration and its later amendments. The inclusion criteria were paroxysmal or persistent AF and a signed informed consent; major exclusion criteria were significant valvular disease, severe left ventricular dysfunction, thyrotoxicosis, and left atrial diameter $>60 \mathrm{~mm}$. Minor exclusion criteria were the absence of sinus rhythm before and after the electrophysiology (EP) procedure, administration of any AADs during the procedure or the need for DC cardioversion during the procedure. Patients were randomly divided in two groups (PVI alone is PVI group, and PVI plus GP ablation is GP group) using the envelope method.

Patients in the PVI group underwent PVI, performed using our standard PVI procedures (see below). Patients in the GP group, underwent PVI, using the same procedures as the PVI group, followed by a GP ablation of presumed GP sites.

\section{Ablation protocol}

All the procedures were done in the morning in the fasting state (overnight fasting). All electrophysiological procedures were performed using the Carto 3 mapping system and the LabSystem Pro (Boston Scientific) EP recording system, which used a decapolar catheter inserted into the coronary sinus (Dynamic, Boston Scientific). Two trans-septal punctures were made (under intracardiac echocardiography (ICE) guidance (AcuNav, Siemens)), one for the circular mapping (Lasso) catheter and the other for the CARTO ablation catheter (ThermoCool Smart-Touch, Biosense-Webster, Inc.). After creating an anatomical CARTO map of the left atrium, the borders of all four pulmonary veins (PVs) were visualized using ICE and marked on the CARTO map. Next, all 4 PVs were isolated using point-by-point radiofrequency ablation $(25-35 \mathrm{~W}$ on the anterior wall, 20-25 W on the posterior wall, maximum temperature $43{ }^{\circ} \mathrm{C}$, contact force $>5 \mathrm{~g}$ ). In the majority of cases, the left veins were isolated by one wide circle without touching the carina between them. The right pulmonary veins were isolated separately. The isolation of each vein was checked for entrance and exit blocks using Lasso catheters. Once the isolation of all PVs was complete, no further ablations were performed in PVI group. In the GP group, after isolation of all pulmonary veins, the ganglionated plexuses were ablated based on GP locations reported by Pokushalov et al. (2013) and Katritsis et al. (2013). In brief, four areas in the left atrial (LA) walls were ablated: (1) superior and posterior to left superior pulmonary vein (LSPV, 1-2 cm outside the ostium of the LSPV and in the area between the superior aspect of the pulmonary vein (PV) antrum and the posterior LA wall), (2) inferior and posterior to the left inferior pulmonary vein (LIPV), (3) anterior to the right superior pulmonary vein (RSPV) (1-2 cm from the RSPV to the supero-anterior LA wall), and (4) below the right inferior pulmonary vein (RIPV, $1-2 \mathrm{~cm}$ inferior to the RIPV). For each GP, at least $5 \mathrm{RF}$ application (20-30 s, 20-25 W) were made, depending on the ablation site. In patients with documented typical flutter, the sheath was withdrawn to the right atrium, a 20-polar hallo catheter was added, and an ablation of the cavotricuspid isthmus was also performed.

\section{Heart rate variability}

Heart rate variability was used as an indicator of autonomic activity in accordance with guidelines for standardization (Heart Rate Variability. Task Force of the European Society of Cardiology the North American Society of Pacing Electrophysiology 1996). Time-domain and frequency-domain indices were calculated from 5-minute ECGs recorded before (on the table in the EP lab just prior to groin puncture) and immediately after the procedure (after all catheters had been withdrawn, although sheaths were left in the inferior vena cava). The 
LabSystem Pro EP recording system (Boston Scientific) was used for signal recording. ECGs from patients were exported to .txt files. These .txt files were prepared in a specific format using appropriate software for further analysis in Kubios HRV (University of Eastern Finland) (Tarvainen et al. 2014). All the analyses were done offline after the procedure, however, all ECG tracings and data were recorded and collected prospectively. All ectopic beats were excluded from analysis and all R-wave detection errors were corrected using manual inspection. The time-domain parameter, i.e. the mean heart rate and the standard deviation of instantaneous heart values, were evaluated. Spectral analysis of electrocardiogram segments was performed using an autoregressive model. The normalized values of the power of the low frequencies (LF) and high frequencies (HF) were investigated. The autonomic balance was assessed using the spectral LF to HF ratio (LF/HF).

\section{Statistical analysis}

Statistical analysis was done using SigmaStat 4.0 software (Systat Software, Inc.). Data were tested for normal distribution using the Shapiro-Wilk test. If the normality test was passed, the differences in the mean value of the parameters were tested using the paired t-test. If the normality test failed, the Wilcoxon Signed Rank test was used. The $\mathrm{P}$ value for significance was 0.05 .

\section{Results}

\section{Patient's and procedural characteristics}

All patients in this study underwent the EP part of the procedure between January 2014 and June 2016. Forty-six patients were enrolled in the study. The PVI group consisted of 26 (10 men, 16 women) patients and the GP group consisted of 20 patients $(12$ men, 8 women). The characteristics of patients and procedures are shown in Table 1. During the procedure, all patients were slightly sedated, using midazolam (approx. 2-5 mg/procedure) and fentanyl (approx. $0.2-0.6 \mathrm{mg} /$ procedure); the sedation was similar in both groups. Fifteen $(57 \%)$ patients in the PVI group and $12(60 \%)$ patients in the GP group were treated by beta-blockers $(\mathrm{p}=0.87)$. One patient in the PVI group $(3.8 \%)$, and one patient in the GP group (5\%) were treated by amiodarone. All other antiarrhythmics were withdrawn 5 half times before ablation.

During the procedure, all patients were slightly sedated, using midazolam $(3.1 \pm 2.1 \mathrm{mg}$ in the PVI group, and $2.7 \pm 1.5 \mathrm{mg}$ in the GP group, $\mathrm{p}=0.7)$ and fentanyl $(380 \pm 172 \mu \mathrm{g}$ in the PVI group, and $413 \pm 200 \mu \mathrm{g}$ in the GP, $\mathrm{p}=0.4)$. Thus, the sedation was similar in both groups. In the PVI group, 7 patients suffered from persistent $\mathrm{AF}$ and 19 patients suffered from paroxysmal $\mathrm{AF}$, and in the GP group, 5 patients suffered from persistent $\mathrm{AF}$ and 15 patients suffered from paroxysmal AF. All patients in both group were in sinus rhythm at the beginning of the procedure and left atrial dimensions were similar in both group.

\section{Vagal responses}

In the PVI group, $96 \%$ ( 25 of 26 patients) had all $4 \mathrm{PVs}$ isolated at the end of the procedure (in 1 patient, only $3 \mathrm{PVs}$ were isolated at the end of the procedure). All patients from the GP group had fully successful PV isolation (i.e. all patients had all $4 \mathrm{PV}$ isolated).

Positive vagal responses (VR, defined by Qin et al. (2016) as a $>20 \%$ decrease in heart rate during sinus rhythm) during ablation were present in $15(58 \%)$ patients in the PVI group and in $12(60 \%)$ patients in GP group. The mean number of VR per patient was $4.5 \pm 2.3$ (range 2-11 VR) in the PVI group. Thirteen patients $(87 \%)$ in PVI group experienced VR during ablation around the LSPV, 6 patients $(40 \%)$ around the LIPV, 3 patients $(20 \%)$ anterior to the RSPV, and 4 patients (27\%) anterior to the RIPV (Fig. 1).

In the GP group, there were $3.8 \pm 2.8$ VRs per patient (range 1-10 VR). The ablation locations where VR was observed, was similar to the PVI group. Eleven patients $(92 \%)$ experienced VR during ablation anterior to the LSPV, 2 patients (17\%) posterior to the LIPV, $5(42 \%)$ anterior to the RSPV, and 7 (58\%) anterior to the LIPV. VR during targeted ablation of GP were only observed in 3 patients $(25 \%)$ in the GP group, all the other VR were observed during the PVI performed prior to GP ablation. Typical areas with vagal responses observed during ablations are shown in Figure 1.

\section{The effect of the ablation on HRV}

Five-minute-long ECG recordings of all patients obtained before and after the EP procedure were analyzed (results are shown in Table 2). The most important parameter among the time-domain results was HR. Heart rate before the procedure was statistically significantly lower in both groups compared to the HR at the end of the procedure (PVI group: before EP $67 \pm 10 \mathrm{bpm}$ vs. after EP $79 \pm 14$ bpm, $p<0.00$ and GP group: $68 \pm 12$ bpm before EP vs. $75 \pm 11$ bpm after $E P, p=0.005$ ). 
Table 1. Characteristics of patients and procedures.

\begin{tabular}{|c|c|c|c|}
\hline & PVI group & GP group & Total \\
\hline \multicolumn{4}{|l|}{ Gender, $n(\%)$} \\
\hline Female & $16(62 \%)$ & $8(40 \%)$ & $24(52 \%)$ \\
\hline Male & $10(38 \%)$ & $12(60 \%)$ & $22(48 \%)$ \\
\hline Age, mean $\pm S . D .^{l}$ & $61 \pm 11$ & $60 \pm 10$ & \\
\hline$B M I^{2}$ & $29.2 \pm 5.5$ & $27.3 \pm 3.9$ & \\
\hline Persistent $A_{F^{2}}$ & 7 & 5 & 12 \\
\hline Long-standing persistent AF & 0 & 0 & 0 \\
\hline Paroxysmal AF & 19 & 15 & 34 \\
\hline \multicolumn{4}{|l|}{ Echocardiography parameters } \\
\hline Ejection fraction $(\%) \pm S . D$. & $62 \pm 3$ & $61 \pm 3$ & \\
\hline Left atrial dimension $(\mathrm{mm}) \pm S . D$. & $38 \pm 5$ & $39 \pm 5$ & \\
\hline \multicolumn{4}{|l|}{ Ablation parameters } \\
\hline Time of the procedure (min) & $216 \pm 42$ & $208 \pm 40$ & \\
\hline Fluoroscopy time (min) & $15 \pm 5$ & $16 \pm 6$ & \\
\hline Left atrial volume according to Carto (ml) & $82 \pm 19$ & $95 \pm 23$ & \\
\hline Total ablation time (min) & $45 \pm 13$ & $48 \pm 7$ & \\
\hline \multicolumn{4}{|l|}{ Ablation time of $G P^{4}$} \\
\hline$L S P V G P(s)$ & - & $260 \pm 79$ & \\
\hline$L I P V G P(s)$ & - & $168 \pm 65$ & \\
\hline$R S P V G P(s)$ & - & $255 \pm 93$ & \\
\hline$R I P V G P(s)$ & - & $153 \pm 85$ & \\
\hline Completed all $P V^{5}$ isolation (\%) & $96 \%$ (25 from 26$)$ & $100 \%$ & \\
\hline Completed box lesion (\%) & - & - & \\
\hline Vagal reaction during ablation & $15(58 \%)$ & $12(60 \%)$ & \\
\hline
\end{tabular}

${ }^{1}$ S.D. - standard deviation, ${ }^{2}$ - BMI - body mass index, ${ }^{3}-\mathrm{AF}$ - atrial fibrillation, ${ }^{4}-\mathrm{GP}$ - ganglionated plexus, ${ }^{5}-\mathrm{PV}$ - pulmonary vein.

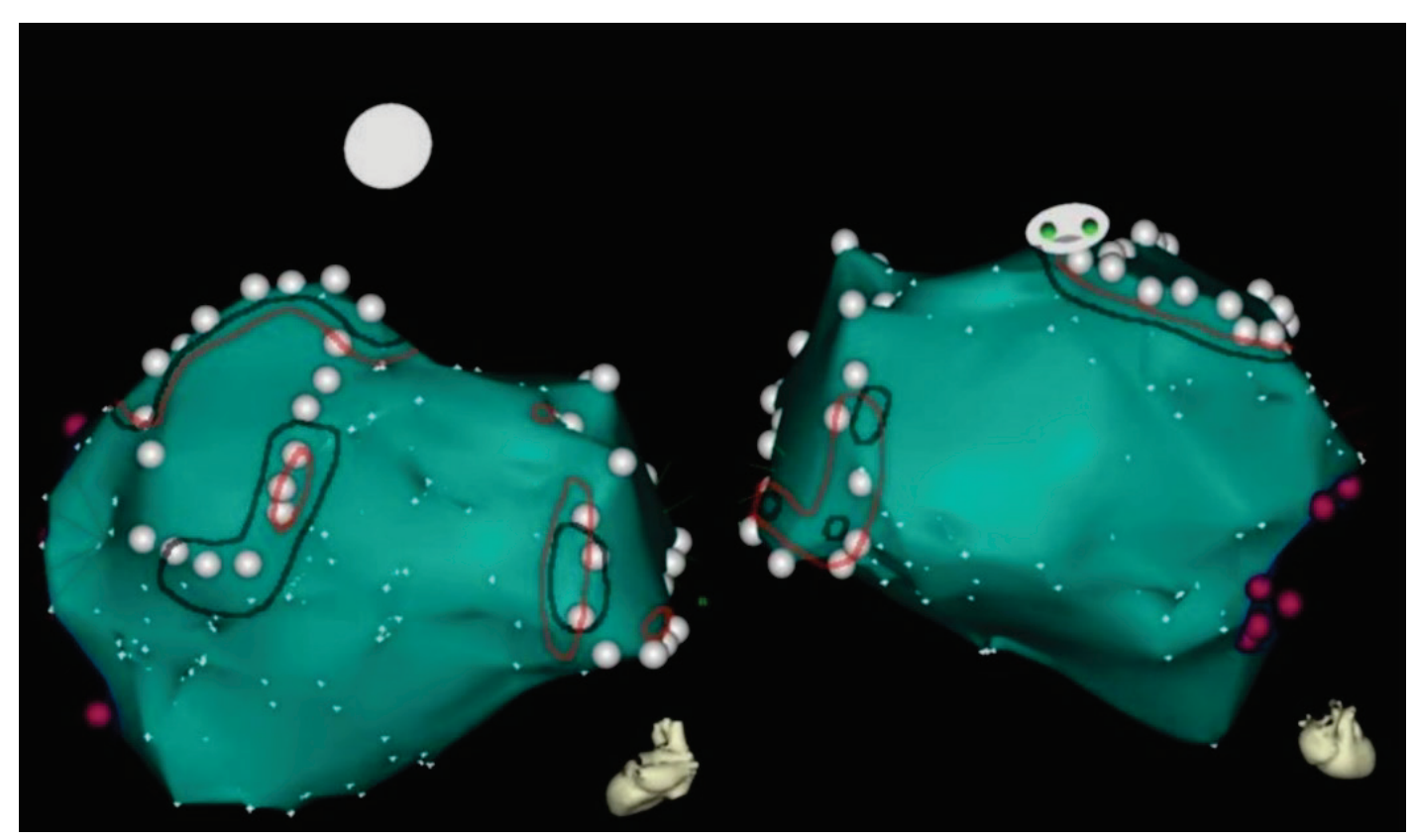

Fig. 1. Typical areas with vagal responses were observed during ablations. The red areas were seen in the GP group and the green areas were seen in the PVI group. 
Table 2. Calculated time and frequency domain parameters.

\begin{tabular}{|c|c|c|c|c|c|c|}
\hline & \multicolumn{3}{|c|}{ PVI group } & \multicolumn{3}{|c|}{ GP group } \\
\hline & Before EP & After EP & $\mathrm{p}$ & Before EP & After EP & $\mathrm{p}$ \\
\hline \multicolumn{7}{|c|}{ Time domain results } \\
\hline Mean $R R^{2}(\mathrm{~ms})$ & $918 \pm 134$ & $784 \pm 149$ & $<0.001$ & $915 \pm 157$ & $823 \pm 125$ & 0.002 \\
\hline$S D N N^{3}(m s)$ & $48 \pm 24$ & $39 \pm 33$ & 0.207 & $58 \pm 39$ & $45 \pm 45$ & 0.305 \\
\hline Mean $H^{4}(1 / \mathrm{min})$ & $67 \pm 10$ & $79 \pm 14$ & $<0.001$ & $68 \pm 12$ & $75 \pm 11$ & 0.005 \\
\hline$S T D^{5} H R(1 / \mathrm{min})$ & $3 \pm 1$ & $3 \pm 3$ & 0.741 & $5 \pm 4$ & $4 \pm 4$ & 0.665 \\
\hline \multicolumn{7}{|c|}{ Frequency domain results $-A R^{6}$ spectrum } \\
\hline \multicolumn{7}{|l|}{$L F^{7}(0.04-0.15 \mathrm{~Hz})$} \\
\hline Peak $(H z)$ & $0.044 \pm 0.006$ & $0.053 \pm 0.027$ & 0.250 & $0.045 \pm 0.009$ & $0.048 \pm 0.022$ & 1 \\
\hline Normalized power (n.u.) & $67 \pm 12$ & $50 \pm 16$ & $<0.001$ & $70 \pm 13$ & $50 \pm 24$ & 0.001 \\
\hline \multicolumn{7}{|l|}{$H F^{s}(0.15-0.4 \mathrm{~Hz})$} \\
\hline $\operatorname{Peak}(H z)$ & $0.186 \pm 0.060$ & $0.210 \pm 0.067$ & 0.214 & $0.175 \pm 0.050$ & $0.205 \pm 0.070$ & 0.06 \\
\hline Normalized power (n.u.) & $32 \pm 12$ & $50 \pm 16$ & $<0.001$ & $30 \pm 13$ & $50 \pm 24$ & 0.001 \\
\hline Total power $\left(\mathrm{ms}^{2}\right)$ & $2,987 \pm 3,384$ & $2,685 \pm 4,250$ & 0.322 & $4,820 \pm 7,739$ & $3,510 \pm 6,940$ & 0.330 \\
\hline$L F / H F^{9}$ & $2.6 \pm 1.6$ & $1.4 \pm 1.7$ & $<\mathbf{0 . 0 0 3}$ & $3.3 \pm 2.6$ & $1.8 \pm 1.9$ & 0.030 \\
\hline
\end{tabular}

${ }^{1}$ - EP - electrophysiology, ${ }^{2}-\mathrm{RR}$ - heart rate interval during sinus rhythm, ${ }^{3}-\mathrm{SDNN}$, standard deviation of consecutive normal-tonormal intervals, ${ }^{4}-\mathrm{HR}$ - heart rate, ${ }^{\mathbf{5}}$ - STD HR - standard deviation of consecutive normal-to-normal beat, ${ }^{6}$ - AR - autoregressive, ${ }^{7}$ - LF - low frequency, ${ }^{8}-\mathrm{HF}$ - high frequency, ${ }^{9}-\mathrm{LF} / \mathrm{HF}$, ratio between low-frequency and high-frequency power.

Regarding the analysis of the frequency-domain, low frequency (LF) and high frequency (HF) parameters were analyzed. The results of peak frequency, HF-, and LF-domain parameters are shown in Table 2. Normalized power in the LF part decreased immediately after the procedure compared to values before the procedure, this was true in both the PVI and GP groups (67 \pm 12 n.u. before and $50 \pm 16$ n.u. after, $p<0.001$ in the PVI group, resp. $70 \pm 13$ n.u. vs. $50 \pm 24$ n.u., $p=0.001$ in the GP group). Additionally, the relative changes were similar in both groups (the increase in HF power was $48 \%$ in the PVI group and $50 \%$ in the GP group). Furthermore, normalized power in the HF part increased after the procedure compared to values before the procedure, and was similar in both groups ( $32 \pm 12$ n.u. before and $50 \pm 16$ n.u. after the procedure, $p<0.0001$ in the PVI group, and $30 \pm 13$ n.u. before and $50 \pm 24$ after the procedure, $\mathrm{p}=0.001$ in the GP group). The $\mathrm{LF} / \mathrm{HF}$ ratio decreased significantly in both the PVI and GP group (2.6 \pm 1.6 before and $1.4 \pm 1.7$ after, $\mathrm{p}<0.003$, and $3.3 \pm 2.6$ before vs. $1.8 \pm 1.9$ after, $\mathrm{p}=0.03$; Table 2, Fig. 2). The decrease in the LF/HF ratio was not significantly different between the two groups.

\section{Discussion}

Surprisingly, in our patients, the PVI performed as a single procedure without touching the presumed GP locations had a similar effect on HRV parameters as PVI plus targeted GP ablation.

GP ablation, with the goal of denervation of the ANS, has been proposed as a promising strategy when added to pulmonary vein isolation (Pappone 2004, Zhang et al. 2012). The autonomic nervous system facilitates genesis and maintenance of atrial fibrillation and atrial denervation effectively reduces AF inducibility (Scherlag et al. 2005). GP ablation (defined as abolishment of VRs during ablation) often occurs as "collateral damage" during PVI. Pappone (2004) reported that vagal responses were observed in $34.3 \%$ of patients during wide circumferential PVIs. VRs were elicited from more than one site in most patients. The most common VR locations were the cranial junction between the left superior PV and LA (95\% patients) and the septal or anterior junction between the right superior PV and LA (25\% patients) (Pappone 2004). Similar results were published Qin et al. (2016). They observed a vagal reaction during PVI in 156 of 479 patients (33\%). VRs were elicited 
during RF ablation on the roof of the LSPV (65.4\%) and the anterior part of the RSPV (44.9\%) (Quin et al. 2016). Our results are in agreement with these observations. In our study, vagal responses were seen in 15 (58\%) patients in the PVI group and in $12(60 \%)$ patients in the GP group. VRs were also seen in more than one site per patient $(4.5 \pm 2.3$ vs. $3.8 \pm 2.8$ in the PVI and GP group, respectively). Vagal reactions were seen in $87 \%$ and $92 \%$ of patients during ablations around the LSPV and in $20 \%$ and $42 \%$ of patients during ablation around the RSPV, in the PVI and GP group, respectively.

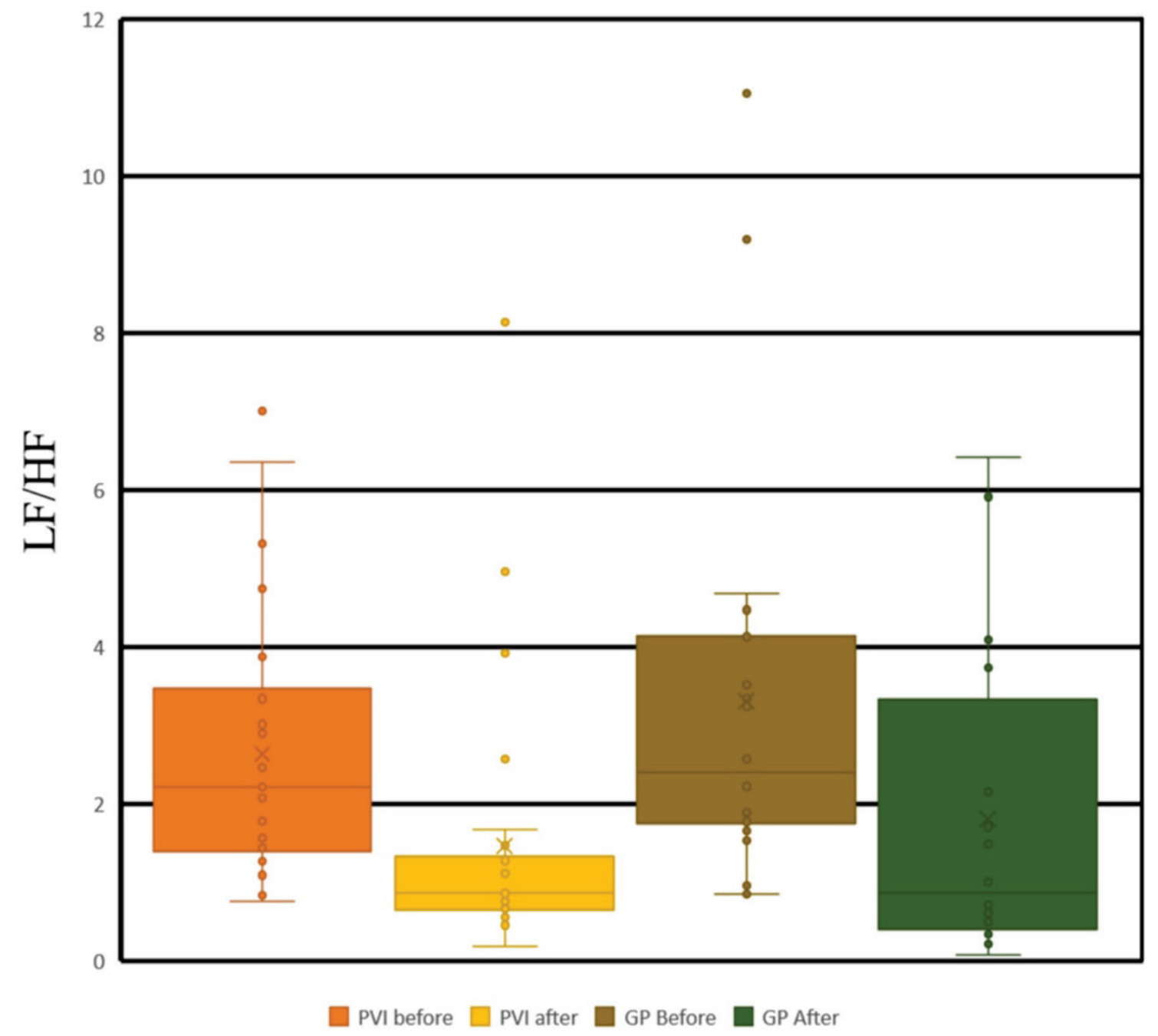

Fig. 2. LF/HF ratios for both groups. There was a significant decrease in the LF/HF ratio in the PVI and GP group (i.e. comparing values pre- and post-procedure).

We hypothesized more vagal responses during anatomical GP ablation. However, vagal responses were only seen in three patients (15\%) in GP group during the GP ablation procedure. On the other hand, in the GP group, vagal responses were present in $70 \%$ of patients during the preceding PVI. Thus, the absence of the vagal responses during GP ablation in the GP group could not be explained by the absence of GPs or vagal responses in general. Since vagal responses were present during the preceding PVI, it could be that the PVI procedure damaged the GPs, which then led to an absence of vagal responses during the GP ablation procedures.

The most accurate technique for locating GPs should be high-frequency (HF) stimulation of presumed GP sites. However, Pokushalov showed that due to the similar locations of GPs in the majority of patients, an anatomical ablation performed empirically (i.e. at presumed GP sites) was associated with similar or even better results compared to ablations driven by GP localization using HF stimulation (Pokushalov et al. 2010). If we look at GP locations, the majority can be 
seen to be quite close to the ostia of the PVs (this is especially true for the upper PVs). Thus, the low incidence of vagal responses during GP ablation in our patients could be explained by GP damage (at least partial damage) that occurred during the immediately preceding pulmonary vein isolation procedure.

Heart rate is modulated by the action of the parasympathetic nervous system (via the vagal nerve) and the sympathetic nervous system (via sympathetic fibers and ganglia). HRV presents a simple, non-invasive method to assess the activity of the autonomic nervous system. As previously demonstrated (Seaborn et al. 2014, Ketels et al. 2008), pulmonary vein isolation is associated with an acute acceleration in heart rate. As expected, we also observed an acute increase in heart rate in both groups of patients. Recently, a decrease in the LF/HF ratio was also described. Seaborn et al. (2014) described a reduction in the LF/HF ratio after PVI $(1.26 \pm 1.0$ before PVI vs. $0.89 \pm 1.05$ one hour after PVI, in patients without AF recurrence and $1.66 \pm 1.7$ before PVI vs. $1.16 \pm 1.24$ after PVI, in patients with AF recurrence). Similarly, a significant decrease in the LF/HF ratio after circumferential and segmental PVI was observed by Wang et al. (2013). According to our calculations, the $\mathrm{LF} / \mathrm{HF}$ ratio, which is used as an accepted parameter of sympatho-vagal balance, decreased significantly during the PVI in the PVI group (2.6 \pm 1.6 before PVI vs. $1.4 \pm 1.7$ after PVI), as well as after GP ablations in the GP group ( $3.3 \pm 2.6$ before vs. $1.8 \pm 1.9$ after). The changes in both groups were very similar, which indicates that endocardial PV isolation performed antrally might be associated with similar changes in HR variability as it is present in additional GP ablations.

Some studies show that HRV changes during PVI, as a marker of autonomic nervous system modification, can predict the success of PVI in terms of arrhythmia - free survival. Yoshida et al. (2009) showed that changes in high frequency power after the segmental pulmonary vein isolation was a significant predictor of atrial fibrillation recurrence. Seaborn et al. (2014) have shown that patients with PAF recurrence after a single circumferential pulmonary vein isolation have HRV attenuated by the procedure only intermittently, whereas patients without PAF recurrence have sustained changes in HRV. Our study was not focused on procedural outcome but HRV recordings could be a potentially important marker for recurrence of atrial arrhythmia.

\section{Conclusions}

Acute vagal responses and autonomic nervous system changes were observed in both the PVI and GP group and in similar fashion. Antral PVI alone itself (i.e. without targeted ablation of GPs) might be associated with GP damage (at least to some degree) and therefore may produce similar effects as GP ablations, relative to the cardiac ANS activity.

\section{Study limitations}

The obvious limitation of the current study is the small number of patients enrolled in the PVI + plus GP ablation group. Drugs administered during the procedure, pain or anxiety could affect the acute results of HRV analysis. Furthermore, ablation of ganglionated plexi were empirical without attempt for confirmation (e.g. high frequency stimulation).

\section{Conflict of Interest}

There is no conflict of interest.

\section{Acknowledgements}

The study was supported by a research grant of the Ministry of Health, Czech Republic, Nr. PROGRES Q38 and by a research grant AZV 16-32478A.

\section{References}

BAUER A, DEISENHOFER I, SCHNEIDER R, ZRENNER B, BARTHEL P, KARCH M, WAGENPFEIL S, SCHMITT C, SCHMIDT G: Effects of circumferential or segmental pulmonary vein ablation for paroxysmal atrial fibrillation on cardiac autonomic function. Heart Rhythm 3: 1428-1435, 2006.

BETTONI M: Autonomic tone variations before the onset of paroxysmal atrial fibrillation. Circulation 105: 2753-2759, 2002.

CALO L, REBECCHI M, SCIARRA L, DE LUCA L, FAGAGNINI A, ZUCCARO LM, PITRONE P, DOTTORI S, PORFIRIO M, DE RUVO E, LIOY E: Catheter ablation of right atrial ganglionated plexi in patients with vagal paroxysmal atrial fibrillation. Circ Arrhythm Electrophysiol 5: 22-31, 2012. 
HEART RATE VARIABILITY: standards of measurement, physiological interpretation and clinical use. Task Force of the European Society of Cardiology and the North American Society of Pacing and Electrophysiology. Circulation 93: 1043-1065, 1996.

JAÏS P, CAUCHEMEZ B, MACLE L, DAOUD E, KHAIRY P, SUBBIAH R, HOCINI M, EXTRAMIANA F, SACHER F, BORDACHAR P, KLEIN G, WEERASOORIYA R, CLÉMENTY J, HAÏSSAGUERRE M: Catheter ablation versus antiarrhythmic drugs for atrial fibrillation: the A4 study. Circulation 118: 2498-2505, 2008.

JANUARY CT, WANN LS, ALPERT JS, CALKINS H, CIGARROA JE, CLEVELAND JR, CONTI JB, ELLINOR PT, EZEKOWITZ MD, FIELD ME, MURRAY KT, SACCO RL, STEVENSON WG, TCHOU PJ, TRACY CM, YANCY CW: Clinical Practice Guideline: 2014 AHA/ACC/HRS Guideline for the management of patients with atrial fibrillation. J Am Col Cardiol 64: 2246-2280, 2014.

KATRITSIS DG, GIAZITZOGLOU E, ZOGRAFOS T, POKUSHALOV E, PO SS, CAMM AJ: Rapid pulmonary vein isolation combined with autonomic ganglia modification: A randomized study. Heart Rhythm 8: 672-678, 2011.

KATRITSIS DG, POKUSHALOV E, ROMANOV A, GIAZITZOGLOU E, SIONTIS GC, PO SS, CAMM AJ, IOANNIDIS JP: autonomic denervation added to pulmonary vein isolation for paroxysmal atrial fibrillation a randomized clinical trial. J Am Col Cardiol 62: 2318-2325, 2013.

KETELS S, HOUBEN R, VAN BEEUMEN K, TAVERNIER R, DUYTSCHAEVER M: Incidence, timing, and characteristics of acute changes in heart rate during ongoing circumferential pulmonary vein isolation. Europace 10: 1406-1414, 2008.

PAPPONE C: Pulmonary vein denervation enhances long-term benefit after circumferential ablation for paroxysmal atrial fibrillation. Circulation 109: 327-334, 2004.

POKUSHALOV E, ROMANOV A, ARTYOMENKO S, TUROV A, SHIROKOVA N, KATRITSIS DG: Left atrial ablation at the anatomic areas of ganglionated plexi for paroxysmal atrial fibrillation. Pacing Clin Electrophysiol 33: 1231-1238, 2010.

POKUSHALOV E, ROMANOV A, KATRITSIS DG, ARTYOMENKO S, SHIROKOVA N, KARASKOV A, MITTAL S, STEINBERG JS: Ganglionated plexus ablation vs linear ablation in patients undergoing pulmonary vein isolation for persistent/long-standing persistent atrial fibrillation: A randomized comparison. Heart Rhythm 10: 1280-1286, 2013.

QIN M, LIU X, JIANG WF, WU SH, ZHANG XD, PO S: Vagal response during pulmonary vein isolation: Re-recognized its characteristics and implications in lone paroxysmal atrial fibrillation. Int $J$ Cardiol 211: 7-13, 2016.

SCHERLAG BJ, YAMANASHI W, PATEL U, LAZZARA R, JACKMAN WM: Autonomically induced conversion of pulmonary vein focal firing into atrial fibrillation. J Am Col Cardiol 45: 1878-1886, 2005.

SEABORN GEJ, TODD K, MICHAEL KA, BARANCHUK A, ABDOLLAH H, SIMPSON CS, AKL SG, REDFEARN DP: Heart rate variability and procedural outcome in catheter ablation for atrial fibrillation. Ann Noninvasive Electrocardiol 19: 23-33, 2014.

TARVAINEN MP, NISKANEN JP, LIPPONEN JA, RANTA-AHO PO, KARJALAINEN PA: Kubios HRV - Heart rate variability analysis software. Comput Methods Programs Biomed 113: 210-220, 2014.

WANG K, CHANG D, CHU Z, YANG Y, GAO L, ZHANG S, XIA Y, DONG Y, YIN X, CONG P, JIA J: Denervation as a common mechanism underlying different pulmonary vein isolation strategies for paroxysmal atrial fibrillation: evidenced by heart rate variability after ablation. ScientificWorldJournal 2013: 569564, 2013.

WILBER DJ, PAPPONE C, AUGELLO G, NEUZIL P, REDDY V, DE PAOLA A, MARCHLINSKI F, NATALE A, MACLE L, DAOUD EG, CALKINS H, HALL B, REYNOLDS MR, VINEKAR C, LIU CY, BERRY SM, BERRY DA: Comparison of antiarrhytmic drug therapy and radiofrequency catheter ablation in patients with paroxysmal atrial fibrillation: A randomized controlled trial. JAMA 303: 333-340, 2010.

YOSHIDA N, YAMADA T, MURAKAMI Y, OKADA T, NINOMIYA Y, TOYAMA J, YOSHIDA Y, TSUBOI N, MUTO Y, INDEN Y, HIRAI M, MUROHATA T: Vagal modification can also help prevent late recurrence of atrial fibrillation after segmental pulmonary vein isolation. Circ J 73: 632-638, 2009. 
ZHANG Y, WANG Z, WANG W, WANG J, GAO M, HOU Y: Efficacy of cardiac autonomic denervation for atrial fibrillation: a meta-analysis. J Cardiovasc Electrophysiol 23: 592-600, 2012. 